\title{
¿CUÁNDO EL DUELO ES PATOLÓGICO Y CÓMO HAY QUE TRATARLO?
}

\author{
Enrique Echeburúa \\ Arantxa Herrán Boix
}

Facultad de Psicologia. Universidad del Pais Vasco

\section{RESUMEN}

El pesar por la muerte de un ser querido es una experiencia que todos los seres humanos sienten. Sin embargo, no todos experimentan las mismas necesidades psicológicas ni tienen los mismos recursos personales y sociales disponibles. $L a$ muerte de una persona allegada es un factor muy estresante, pero muchas personas se muestran resistentes emocionalmente para superar este trance. La pena mórbida se produce cuando la persona experimenta durante el proceso del duelo emociones negativas (tristeza, ira, culpa, confusión) muy intensas y duraderas que interfieren negativamente en su vida cotidiana. Entre el 10 y el $20 \%$ de las personas afectadas por una pérdida pueden necesitar ayuda profesional. En estos casos el tratamiento debe darse de forma temprana para evitar la cronificación de los sintomas. La lamentación dirigida, la exposición y la entrenamiento en competencias contribuyen a aliviar los sintomas depresivos y a reconstruir la autoestima y las creencias sobre las habilidades personales. Los grupos de autoayuda pueden ser una ayuda complementaria, sobre todo

Correspondencia: Facultad de Psicología. Universidad del País Vasco. Avda. de Tolosa, 70 20018 San Sebastiann (Spain).E-mail: enrique.echeburua@ehu.es; aherranboix@hotmail.com 
cuando las personas afectadas por la pena mórbida se encuentran aisladas socialmente. Se comentan sugerencias para la investigación futura en este campo.

Palabras clave: DUELO, PENA MÓRBIDA, VULNERABILIDAD, RESISTENCIA, LAMENTACIÓN DIRIGIDA, TRATAMIENTO.

\section{SUMMARY}

Bereavement is a common and natural experience that we all share, but not all have the same personal and social resources available or have the same experiences and needs. Bereavement is very stressful but many people manage their losses with considerable resiliency. Morbid grief refers to the negatively severe affective or emotional responses (confusion, sadness, anger and guilt) that some people can experience during the bereavement process and that seriously alter daily routines. Between 10 and $20 \%$ will need some kind of professional help with their bereavement adjustments. Intervention need to be available early in the bereavement process because the early adjustments will influence outcomes much later. Guided mourning, exposure and behavioural competency intervention may be useful to cope with depressive symptoms and to rebuild the self-esteem and beliefs about personal competence. Selfhelp support groups may be and adjunctive intervention, mostly when people affected by morbid grief are socially isolated. Suggestions for further research in this area are commented upon.

Key words: BEREAVEMENT, MORBID GRIEF, PREDICTORS OF BEREAVEMENT ADJUSTMENTS, GUIDED MOURNING, EXPOSURE, TREATMENT. 


\section{INTRODUCCIÓN}

La muerte de un ser querido es una experiencia amarga. El duelo es el conjunto de reacciones de tipo físico, emocional y social que se producen por el fallecimiento de una persona próxima y que pueden oscilar desde un sentimiento transitorio de tristeza hasta una sensación de desgarro y de desolación completa, que, en los casos más graves, puede durar años e incluso toda la vida (Echeburúa, 2004). El proceso de duelo supone una reacción adaptativa ante la muerte de un ser querido, que obliga al sujeto afectado a rehacer su vida desde una perspectiva diferente. En realidad, el duelo es el proceso de asimilar una pérdida (Pérez Cuesta y Nicuesa, 1999). Los recursos desplegados para superar el duelo constituyen un reflejo del instinto de supervivencia para hacer frente a las dificultades de la vida.

Cada ser humano reacciona de manera diferente ante una pérdida, sin que exista una pauta inamovible de cómo una persona tiene que manifestar y afrontar su dolor. Es más, además de las diferencias entre personas, hay variaciones en una misma persona en las distintas pérdidas experimentadas. Al ser cada persona fallecida única e irrepetible, así como las relaciones interpersonales que se establecen con ellas, el superviviente nunca vivenciará de la misma forma la muerte de dos allegados. Por lo tanto, cada proceso de duelo será, en cierto modo, siempre diferente (Bonanno y Kaltman, 2001).

Tras la muerte de un ser querido por causas naturales, las respuestas normales de la mayoría de la gente son la aceptación y la añoranza por el fallecido. Y las emociones negativas propias del duelo suelen disminuir hacia los seis meses de la pérdida. En cualquier caso, las respuestas adaptativas puestas en marcha tras la muerte de un ser querido son muy variables de unos casos a otros. No hay que pasar necesariamente por un período de intenso sufrimiento ni seguir un ciclo predeterminado (pérdida, depresión, proceso de elaboración y resolución final) (Avia y Vázquez, 1998).

Afrontar el duelo es un proceso psicológico, no psicopatológico, porque la muerte forma parte de la vida y la vida no es una enfermedad. Estar transitoriamente triste (una situación emocional normal) no significa estar deprimido (un cuadro clínico). Ello quiere decir que, en 
la mayor parte de los casos, la superación del duelo no requiere un tratamiento psicológico. Los recursos psicológicos de la persona, el paso del tiempo, el apoyo familiar y social y la reanudación de la vida cotidiana suelen ser suficientes para asimilar la pérdida y readaptarse a las nuevas circunstancias.

El problema psicopatológico se plantea cuando la persona experimenta un sufrimiento insufrible, cuando se encuentra clínicamente deprimida y cuando no cuenta con recursos psicológicos suficientes o sus estrategias de afrontamiento son erróneas. En estos casos el superviviente muestra una incapacidad para adaptarse a la nueva situación planteada tras la pérdida. Es entonces cuando la persona muestra un duelo patológico y necesita ayuda externa para salir adelante y readaptarse a la vida cotidiana.

En este trabajo se analiza el problema del duelo en los adultos. Las características peculiares del ciclo evolutivo en niños y ancianos requieren un tratamiento diferenciado de este problema.

\section{DUELO NORMAL Y DUELO PATOLÓGICO}

El duelo por la muerte de un ser querido puede manifestarse en forma de síntomas somáticos (pérdida de apetito, insomnio, síntomas hipocondríacos, cefaleas, etcétera) y psicológicos (pena y dolor, fundamentalmente). Puede haber también una disminución de las defensas del organismo, lo que aumenta la vulnerabilidad del sujeto a padecer enfermedades físicas.

\section{Duelo normal}

La tristeza -y la ansiedad en segundo lugar- es el sentimiento más común tras la muerte de un ser querido, junto con el temor a afrontar la soledad. A veces pueden aparecer también sentimientos de culpa, por no haber realizado todo lo posible para evitar el fallecimiento, por no haber hecho al difunto lo suficientemente feliz en vida o incluso por experimentar una sensación de alivio tras la muerte (especialmente, después de una enfermedad prolongada que ha requerido de una asistencia constante o de la desaparición de una persona que ha ejercido un dominio despótico sobre el superviviente). 
También suele disminuir el interés del sujeto por el mundo exterior, sobre todo en lo que recuerda a la persona fallecida. $Y$ puede sobrevenir el desinterés por el trabajo y por encontrar nuevas relaciones, asi como la apatía y la falta de esperanza y de ilusión ante el futuro.

La intensidad de estos síntomas depende de la personalidad del sujeto, de la intensidad del lazo que le unía al fallecido y de las circunstancias de la pérdida, entre otros aspectos. Lo normal y esperable, cuando se utilizan unas estrategias de afrontamiento positivas (tabla 1), es una que estos síntomas o emociones negativas remitan paulatinamente en un plazo de 6 meses a un año.

Tabla 1. Estrategias de afrontamiento positivas

- Aceptación del hecho y resignación

- Experiencia compartida del dolor y de la pena

- Reorganización del sistema familiar y de la vida cotidiana

- Reinterpretación positiva del suceso (hasta donde ello es posible)

- Establecimiento de nuevas metas y relaciones

- Búsqueda de apoyo social

- Implicación en grupos de autoayuda o en ONG

Hay que tener en cuenta que las primeras reacciones no marcan necesariamente la evolución del duelo. Algunas personas, en las primeras semanas, tienen una respuesta emocional ligera y, sin embargo, experimentan una intensificación y cronificación del duelo posterior. Otras, en cambio, se expresan con un sufrimiento intenso inicial, para luego ir superando el duelo más fácilmente, lo que suele ser la reacción más frecuente (Echeburúa, 2004).

\section{Duelo patológico}

Cuando las reacciones emocionales son muy intensas e impiden el funcionamiento en la vida diaria, la duración de la reacción es 
anormalmente larga (dura más allá de un año) y aparecen síntomas inhabituales (por ejemplo, alucinaciones, referidas a visiones o voces del fallecido, ideas delirantes o pensamientos suicidas recurrentes), se puede hablar de duelo patológico (Femández-Montalvo y Echebunua, 1997; Parkes, 1972; Worden, 1998). La pena mórbida es la intensificación del duelo hasta un punto en que la persona niega la pérdida, se siente desbordada, recurre a conductas desadaptativas 0 permanece inacabablemente en este estado, sin mostrar avances en el proceso de resolución del duelo (Horowitz, Wilner, Marmar y Krupnick, 1980) (tabla 2).

Tabla 2. Estrategias de afrontamieto negativas

- Anclaje en los recuerdos y planteamiento de preguntas sin respuesta

- Sentimientos de culpa

- Emociones negativas de ira o de odio

- Abandono del cuidado personal

- Aislamiento social

- Consumo excesivo de alcohol o drogas

- Abuso de medicinas

El duelo patológico se puede manifestar a nivel psicológico en forma de autorreproches (por ejemplo, por no haber hecho lo suficiente por el difunto, incluso si han extremado de modo admirable sus atenciones durante su última enfermedad), de pensamientos de muerte persistentes o de una irritabilidad inhabitual contra terceras personas (médicos, sacerdotes, yerno o nuera, etcétera). A veces, la tristeza patológica puede complicarse con otros síntomas, como el aislamiento social, la falta de cuidado personal, el enlentecimiento psicomotor acusado, el consumo abusivo de alcohol o de psicofármacos, etcétera. A nivel físico, pueden surgir alteraciones de la salud persistentes: 
trastornos del sueño y del apetito, fatiga, dolores musculares, conductas hipocondríacas, estreñimiento, cefaleas, etcétera, lo que les lleva a consultar con mucha mayor frecuencia de lo habitual al médico de familia. Todo ello es una somatización de la depresión existente (Alario, 1996; Hinton, 1974).

En las fases iniciales del duelo pueden predominar los síntomas físicos. Sin embargo, con el transcurso de los meses estos síntomas pueden declinar y hacerse más frecuentes los síntomas psicológicos: desesperanza, desvalorización, ira, deseos de morir, etc. (Clayton, 1985).

El duelo patológico se manifiesta en forma de depresión, pero a veces puede revestir también la forma de un trastorno de ansiedad o de somatización. En cualquier caso, la pena mórbida no afecta a más del $10 \%-20 \%$ de los supervivientes de una persona fallecida.

La detección del duelo patológico se lleva a cabo por dos vías: la búsqueda de ayuda terapéutica por este problema o la consulta por algún otro tipo de problema médico o psiquiátrico. Esta segunda vía es mucho más frecuente (Lazare, 1979).

\section{CARACTERÍSTICAS DEL DUELO EN FUNCIÓN DEL TIPO DE PÉRDIDAS}

Además del equilibrio emocional del superviviente y del apoyo familiar y social recibido, la evolución de un duelo normal a un duelo patológico depende de diversas circunstancias, tales como la vinculación con la persona fallecida, el tipo de muerte o las circunstancias que rodean la pérdida.

Es decir, la muerte de un ser querido explica los daños provocados por el primer golpe. Pero es el significado que ese suceso tiene en la persona lo que puede explicar los efectos más o menos devastadores del segundo golpe, que es lo que realmente provoca el duelo patológico.

\section{Vinculación con la persona fallecida}

Las muertes más impactantes y las que tienen una mayor resonancia afectiva para el superviviente son las referidas a las personas más próximas de su entorno familiar o de relación: un hijo, la pareja, un padre, un hermano, etc. 
La pérdida de un ser querido puede ser el factor más estresante en la vida de una persona, especialmente si se produce de forma imprevista y se trata de un hijo. El proceso de aceptación de su muerte es el más difícil de todos. La muerte de un hijo es un hecho antinatural, que produce una inversión del ciclo biológico normal. Ni siquiera hay un término para describir al superviviente, como lo hay en el caso de la pérdida de un padre (huérfano) o de un marido (viuda). Alrededor de un $20 \%$ de los padres que pierden a un hijo no llegan a superarlo nunca (Pérez Trenado, 1999).

El dolor y la desesperación por la ausencia se reactivan en determinadas fechas: cumpleaños de sus hijos, Navidades, aniversarios de su muerte, etcétera. El estrés que causa la pérdida de un hijo (el fantasma de la habitación vacía) es tan intenso que provoca con frecuencia conflictos en la relación entre los padres. A menudo, un cónyuge sufre más que otro y eso genera acusaciones, culpabilidad y rabia.

La pérdida de la pareja puede suponer un choque emocional muy intenso, sobre todo cuando el superviviente dependía emocionalmente de ella, tenía un estatus social en función de ella y carece de habilidades - de salud para readaptarse a la nueva situación y sobrevivir emocionalmente por sí solo (el síndrome del corazón roto) (Alper y Liberman, 2001). En otros casos, por el contrario, la persona puede readaptarse positivamente cuando adquiere una autonomía de la que carecía, se implica en nuevas actividades y descubre en ella unas potencialidades de las que no era consciente.

\section{Tipo de muerte}

Existen ciertos tipos especiales de muerte (homicidios, suicidios, muertes repentinas) que pueden requerir un esfuerzo adicional para integrar un proceso de duelo adecuado y superar con éxito el mismo.

El impacto emocional mayor de una muerte en un superviviente es cuando ésta es prematura, inesperada o trágica. Es el caso, por ejemplo de las enfermedades cortas y repentinas o de las muertes súbitas, evitables y que afectan a personas jóvenes, como ocurre en los accidentes de tráfico. Asimismo una muerte precedida de una enfermedad dolorosa, no asumida por el paçiente, puede dificultar la superación del duelo del superviviente. 
Las muertes violentas, ya sean por asesinato o por suicidio, constituyen un apartado especial en la recuperación de los supervivientes. En concreto, el dolor de los padres es mucho más intenso cuando ha habido una doble victimización (por ejemplo, en el caso de una joven violada y asesinada), cuando el agresor no ha sido detenido, cuando de algún modo se atribuye lo ocurrido al estilo de vida de la victima (a una vida licenciosa, por ejemplo), cuando no ha aparecido el cuerpo de la víctima o cuando, como ocurre habitualmente en el caso de un suicidio o de la desaparición de un menor, los padres pueden sentirse responsables de lo sucedido.

\section{VULNERABILIDAD Y RESISTENCIA AL DUELO PATOLÓGICO}

Las diferentes reacciones ante el fallecimiento de un ser querido permiten vislumbrar la existencia de personalidades vulnerables y de personalidades resistentes. En concreto, la experimentación de un duelo patológico depende de una serie de factores (Echeburúa y Corral, 2001):

1. Inestabilidad emocional previa del paciente. En concreto, las personas con antecedentes de depresión o de trastornos de ansiedad, con estilos de afrontamiento inadecuados o con características problemáticas de personalidad (dependientes emocionalmente, obsesivas, acomplejadas, etcétera) corren un mayor riesgo de derrumbarse emocionalmente y de sufrir un duelo patológico en el caso del fallecimiento de una persona próxima emocionalmente. Asimismo las personas con una salud delicada son las que tienden a caer más fácilmente enfermas durante el duelo (Clayton, 1985).

2. Experiencia negativa de pérdidas anteriores. Es más probable sufrir un duelo patológico cuando una persona ha experimentado muchas o recientes pérdidas y cuando la reacción ante las anteriores ha sido particularmente intensa o duradera.

3. Variables psicosociales, como estar en una situación económica precaria, hacerse cargo de niños pequeños, no tener posibilidad de rehacer la vida, contar con una familia muy reducida, etcétera. Las reacciones de carácter grave ante una pérdida tienen lugar frecuentemente entre las mujeres. Por otra parte, los niños de 3 a 5 
años y los adolescentes, así como los viudos solos y las viudas con niños pequeños y sin apoyo social, constituyen un factor de riesgo importante.

Evidentemente muchos de los factores de riesgo pueden ser considerados de modo inverso como factores protectores. Es el caso del apoyo familiar, de la ocupación laboral o de la ausencia de dificultades económicas. Ya en un plano más psicológico, los sentimientos de utilidad, el control emocional, un estilo de vida equilibrado, un mundo interior rico, unas aficiones gratificantes, la expresión de sentimientos y la ausencia de patologías previas facilitan la recuperación del duelo. Las creencias religiosas y la capacidad para encontrar un sentido a la vida han mostrado asimismo su eficacia como protectores (Barreto y Soler, 2004; Echeburúa, Corral y Amor, 2005) (tabla 3).

Tabla 3. Personalidades resistentes al estrés

- Control de las emociones y valoración positiva de uno mismo

- Estilo de vida equilibrado

- Apoyo social y participación en actividades sociales

- Implicación activa en el proyecto de vida (profesión, familia, actividades de voluntariado, etc.)

- Afrontamiento de las dificultades cotidianas

- Aficiones gratificantes

- Sentido del humor

- Actitud positiva ante la vida

- Aceptación de las limitaciones personales

- Vida espiritual 
Por lo que se refiere a las personas mayores, la autoestima positiva, las estrategias de afrontamiento adecuadas, las relaciones familiares y sociales y una buena salud protegen al superviviente y hacen menos probable la aparición de un duelo patológico (Barbero, 2006).

\section{¿CUÁNDO ES NECESARIO EL TRATAMIENTO?}

Hay personas que han sufrido una pérdida y que, sin embargo, no necesitan una ayuda psicológica o farmacológica. El equilibrio psicológico previo, el transcurso del tiempo, la atención prestada a los requerimientos de la vida cotidiana y el apoyo familiar y social contribuyen muchas veces a digerir la ausencia. De este modo, estas personas, aun con sus altibajos emocionales y con sus recuerdos dolorosos, son capaces de trabajar, de relacionarse con otras personas, de disfrutar de la vida diaria y de implicarse en nuevos proyectos.

Por el contrario, otras personas se encuentran atrapadas por la pérdida sufrida, no recuperan sus constantes biológicas en relación con el sueño y ol apetito, viven atormentadas con un sufrimiento constante, tienen dificultades para controlar sus emociones y sus pensamientos, se aíslan socialmente y se muestran incapaces de hacer frente a las exigencias de la vida cotidiana, adoptando a veces conductas contraproducentes (beber en exceso, comer más o menos de la cuenta, automedicarse, etcétera). Son estas personas las que, al sentirse desbordadas por el dolor, requieren una ayuda específica psicológica y, en algunos casos, también farmacológica (tabla 4).

Tabla 4. Criterios para la búsqueda de ayuda profesional

- Cuando las reacciones psicológicas (pensamientos, sentimientos o conductas) perturbadoras duran más de 4 a 6 semanas.

- Cuando hay una interferencia negativa grave en el funcionamiento cotidiano (familia, trabajo o escuela).

- Cuando una persona se siente incómoda con sus pensamientos, sentimientos o conductas o se siente desbordada por ellos. 
En resumen, el superviviente de una pérdida sólo debe buscar ayuda terapéutica cuando las reacciones psicológicas perturbadoras duran más de 4 a 6 semanas, cuando hay una interferencia negativa grave en el funcionamiento cotidiano (familia, trabajo o escuela) o cuando la persona se siente desbordada por sus pensamientos, sentimientos o conductas.

\section{ESTRATEGIAS TERAPÉUTICAS}

Los tipos de intervención recomendados para los pacientes que han elaborado un duelo patológico son tanto el tratamiento individual como el grupal y/o familiar, sin descartar en algunos casos la combinación de ambas modalidades. La primera de ellos favorece la individualización de la intervención y la adaptación específica a las conductas problemáticas de la persona afectada. La intervención grupal y/o familiar optimiza los recursos y los resultados al brindar apoyo social, facilitar un espacio de encuentro donde expresar pensamientos y emociones, normalizar las manifestaciones de duelo, romper el aislamiento y ofrecer modelos entre iguales. En ocasiones puede ser útil combinar ambas formas de tratamiento para obtener las ventajas de cada una de ellas (Barreto y Soler, 2004).

Los grupos de autoayuda pueden desempeñar un papel importante en los casos de duelo complicado, especialmente cuando se trata de la muerte de un hijo, de un suicidio, de un asesinato o de un atentado terrorista. En algunos casos estos grupos tienen un carácter balsámico en los supervivientes, sobre todo cuando éstos cuentan con una red de apoyo familiar y social pobre, y ayudan a las víctimas a encarar el presente y el futuro. Hay otras personas, sin embargo, para las que estos grupos pueden resultar contraproducentes porque el dolor de otras víctimas les reaviva su propio dolor y les impide afrontar adecuadamente el futuro.

\section{Objetivos terapéuticos}

El objetivo de la intervención no es olvidar al ser querido muerto, sino recolocar al fallecido en el espacio psicológico biográfico del superviviente. Es decir, se trata de recordar al ser querido sin que este recuerdo bloquee el compromiso del superviviente con la vida. 
Más en concreto, las metas del tratamiento son reducir la tristeza y la ansiedad, aumentar la autoestima y disminuir el aislamiento social, todo ello encaminado a mejorar la calidad de vida del paciente y a prevenir la cronificación del trastorno (Rando, 1993).

Se pueden distinguir cinco metas fundamentales en la terapia del duelo patológico, a las que podría atribuirse el éxito de la misma (Echeburúa, 2004; Ruiz y Cano, 2002):

1. Facilitar la expresión de los sentimientos inhibidos hacia el fallecido y el relato de las relaciones habidas con él.

2. Fomentar la expresión de las circunstancias que condujeron a la pérdida.

3. Lograr, a partir de los dos puntos anteriores, la activación cognitiva del procesamiento de informaciones previamente excluidas.

4. Centrar la terapia en la solución de los problemas cotidianos y en la readaptación a la vida diaria.

5. Proyectar al sujeto hacia el futuro de forma gradual, implicándole en la realización de actividades gratificantes.

El ritmo personal del superviviente puede ser variable de unos casos a otros y debe abordarse con la flexibilidad necesaria (Barreto y Soler, 2004).

\section{Vías de intervención}

Los enfoques clínicos utilizados han oscilado entre un modelo más psiquiátrico, orientado genéricamente al tratamiento de los síntomas (depresión, ansiedad, alteraciones del sueño, etcétera), y un acercamiento más psicoterapéutico, orientado específicamente al tratamiento del duelo en sí mismo.

El proceso terapéutico puede dividirse en diversas fases (Echeburúa, 2004; Echeburúa, Corral y Amor, 2001; Raphael, 1977. 1983; Ruiz y Cano, 2002):

1. Explorar la relación del paciente con el fallecido, lo que supone alentarle a que hable de la forma en que ha vivido la pérdida y de las atribuciones que haya podido realizar al respecto. De lo que se trata es de resituar la imagen del fallecido en su biografía, evitando imágenes excesivamente idealizadas $u$ hostiles. En este contexto se analizan las emociones negativas suscitadas (tristeza, culpa, ira, etcétera) y 
se facilita el procesamiento de la información adecuado, introduciendo las reatribuciones correspondientes.

2. Interrumpir la cadena de conductas que anclan al paciente al pasado y le impiden romper amarras con situaciones irreversibles. Por ejemplo, se trata de programar la interrupción gradual de las visitas al cementerio (sólo cuando éstas tienen una alta frecuencia), así como de impedir al paciente recrearse en un pasado de añoranza (ver fotos del fallecido continuamente, hablar de él de forma constante) o de mantener conductas supersticiosas (no utilizar la silla en donde se sentaba, mantener toda su ropa en el armario, etcétera).

3. Utilizar la técnica de la lamentación dirigida (Mawson, Marks, Ramm y Stern, 1981; Sireling, Cohen y Marks, 1988), es decir, exponer cognitivamente al paciente a recuerdos vivenciales en relación con la persona fallecida (sólo cuando hay conductas intensas de evitación cognitiva y bloqueo emocional, con respuestas de reexperimentación en forma de pesadillas o de pensamientos invasivos). La evocación de recuerdos, la lectura de cartas o la presentación de un álbum de fotografías pueden facilitar a veces este tipo de exposición cuando hay una fuerte inhibición emocional. El paciente debe describir repetidamente este tipo de situaciones hasta que disminuya el malestar. El mecanismo subyacente es que la presentación repetida de los estímulos que provocan dolor genera un debilitamiento de la respuesta emocional condicionada (la tristeza). En otros casos la exposición puede ser en vivo y referirse a conductas evitadas por la relación que se establece con la persona fallecida y que, sin embargo, tienen un carácter gratificante: acudir al cine, viajar, ir a casa de unos amigos, etcétera (Echeburúa y Corral, 1997; Echeburúa, Corral, Sarasua y Zubizarreta, 1996).

4. Facilitar la autoaceptación de las limitaciones personales (defectos personales, errores cometidos, etcétera) y enfatizar los aspectos positivos.

5. Atender a los problemas cotidianos planteados por el paciente y facilitar la readaptación a la vida diaria. En este sentido, atender a la salud, prestar atención a la relación familiar y social, facilitar la adaptación al trabajo y vigilar de cerca los hábitos de vida se constituyen en objetivos preferentes.

6. Programar actividades gratificantes gradualmente, especialmente aquellas que implican una relación social, y facilitar el establecimiento 
de metas futuras. Todo lo que suponga sacar al paciente de su caparazón y hacerle sentirse útil ante sí mismo y ante los demás contribuye a su recuperación.

\section{INDICADORES DE RECUPERACIÓN}

Una primera señal de recuperación básica es que la persona recobre las constantes biológicas en relación con el sueño y el apetito. Asimismo un indicador positivo del proceso de mejoría del paciente es cuando reaparece la expresión verbal de los sentimientos y se pone orden en el caos de las imágenes y recuerdos de la pérdida sufrida. A veces, y más allá de las palabras, la reaparición de expresiones de afecto gestuales, como sonrisas, o físicas, como abrazos o besos, es una señal de mejoría.

La implicación en conductas gratificantes, el establecimiento de metas concretas, la reanudación de la vida social o la participación en actividades de voluntariado denotan la recuperación de la persona porque todo ello contribuye a aumentar la autoestima y a imprimir un significado a su vida.

En resumen, el alivio de los síntomas, una experiencia subjetiva de mayor bienestar y el cambio en el estilo de vida están estrechamente asociados a la superación del duelo patológico. En definitiva, el duelo se asimila cuando la persona, aun con dolor, es capaz de integrar la pérdida como algo pasado que forma parte de su historia personal, sin la presencia excesiva de emociones negativas (como rabia, culpa o tristeza profunda) y sin sentirse abrumada por los recuerdos. Se ha conseguido integrar la memoria del fallecido en la vida del superviviente y se es capaz de evocar los recuerdos de las experiencias positivas vividas junto a la persona fallecida. En estos casos la persona puede vivir con normalidad el día a día, disfruta con la vida cotidiana, utiliza unas estrategias de afrontamiento positivas y establece metas futuras (Latiegui, 1999; Lee, 1995; Morganett, 1995; Worden, 1998).

Hay veces, sin embargo, en que el duelo se cronifica. El factor más problemático para la recuperación del superviviente es la inestabilidad emocional anterior, la dependencia excesiva de la pareja o la existencia de actitudes hipocondríacas. Son también indicadores negativos relevantes haber sufrido pérdidas anteriores próximas 0 
acumuladas, tener una salud delicada y haber mostrado una mala capacidad de adaptación a diferentes situaciones adversas en la vida anterior. Pero, en general, la evolución de la recuperación depende de las estrategias de afrontamiento utilizadas para superar el duelo. En concreto, el pronóstico es más sombrío cuando la persona, en lugar de encarar el problema adecuadamente, adopta estrategias de afrontamiento negativas (como beber alcohol en exceso, automedicarse con tranquilizantes, no expresar las emociones o volcarse en el trabajo de una forma compulsiva), mantiene una baja laboral indefinida o se refugia en el pasado. Otras veces la evolución negativa depende de la presencia de sentimientos intensos de culpa o de ira o de la aparición de circunstancias vitales adversas (abandono de los hijos, pérdida de empleo, etcétera).

\section{CONCLUSIONES}

El dolor por la muerte de un ser querido es un fenómeno habitualmente transitorio, forma parte de la vida misma y no suele ser un problema de significación clínica. En general, cuanto más afecte esa pérdida a la vida diaria del sujeto y cuanto más rápida e imprevista sea la muerte, mucho mayor será el impacto emocional.

No existe una receta mágica sobre la manera de afrontar esta experiencia amarga, pero sí hay ciertas directrices que pueden facilitar este trance. En primer lugar, cada persona necesita su propio tiempo y va a sobrellevar el duelo a su manera, de una forma que no es necesariamente intercambiable con la de otras personas. $Y$ en segundo lugar, el paso del tiempo, la expresión de las emociones sentidas, el apoyo social y familiar, la reanudación de la vida cotidiana y la implicación en actividades gratificantes suelen ser suficientes para superar el malestar emocional. Aceptar el sufrimiento y sobreponerse a él es algo muy positivo. Después de todo, la esperanza y el espíritu de superación forman parte del instinto de conservación y de supervivencia del ser humano (Rojas Marcos, 2002).

Hay algunos casos, sin embargo, en que por diversas circunstancias personales (una personalidad vulnerable, la acumulación de pérdidas anteriores, etcétera), situacionales (una muerte brusca o violenta, el fallecimiento de un hijo, etcétera) o sociales 
(la falta de apoyo requerida) el malestar emocional por la muerte de un ser querido se convierte en un duelo patológico que genera un profundo malestar emocional y que interfiere negativamente en la vida de la persona. Es en estos casos cuando se requiere una intervención profesional psicológica y/o médica (Echeburúa, 2004).

Los resultados terapéuticos obtenidos hasta la fecha son muy variables de unos casos a otros. La lamentación dirigida y la exposición en vivo a las situaciones evitadas han conseguido buenos resultados en el tratamiento de la pena mórbida, si bien este método reduce más la evitación de los aspectos de la tristeza que el hundimiento del ánimo. Hay que esclarecer mejor las relaciones entre depresión, pena y evitación de las señales relacionadas con los fallecidos (FernándezMontalvo y Echeburúa, 1997). Queda asimismo por conocer el pertil diferencial de las personas que pueden beneficiarse de los grupos de autoayuda o el papel que puede desempeñar la donación de los órganos del fallecido en la superación del duelo por la pérdida de un ser querido.

El duelo acaba cuando la persona ya no necesita reactivar el recuerdo de la pérdida del fallecido con una intensidad exagerada en la vida diaria (siendo capaz de pensar en el fallecido sin sentirse abrumado), cuando se recupera el sentido de la vida y cuando la persona se siente esperanzada y capaz de disfrutar de nuevo de las pequeñas cosas de la vida. Los escenarios de la alegría son, básicamente, las relaciones íntimas con otras personas, el trabajo 0 las ocupaciones y las actividades de ocio, así como el sentido del humor (Rojas Marcos, 2001; Savater, 1998).

Un reto de futuro es detectar de forma temprana a las personas realmente necesitadas de tratamiento, establecer programas eficaces protocolizados de intervención para personas afectadas por un duelo patológico e integrar los recursos terapéuticos existentes (Centros de Salud Mental, Grupos de Autoayuda, asistencia privada, etcétera). Otro reto de interés es abordar el problema del duelo de forma diferenciada según las peculiaridades del ciclo evolutivo (infancia, tercera edad, etcétera).

Un tratamiento psicológico requiere siempre el establecimiento de una relación de empatía, de confianza básica, entre el paciente y el terapeuta, pero este requisito es especialmente importante en el caso 
del duelo patológico. Asimismo hay que ser especialmente respetuoso con el sistema de creencias de la persona, lo que supone una actitud de neutralidad por parte del terapeuta.

\section{REFERENCIAS BIBLIOGRÁFICAS}

Alario, S. (1996). Pena mórbida. En Muerte y neurosis. Valencia. Promolibro.

Alper, S. y Liberman, D. (2001). Una nueva perspectiva del duelo. Experiencia transformadora. Campo Grupal, 28.

Avia, M.D. y Vázquez, C. (1998). Optimismo inteligente. Madrid. Alianza Editorial.

Barbero, J. (2006). La muerte de un ser querido. Duelo y adaptación en las personas mayores. Madrid. Portal Mayores.

Barreto, M.P. y Soler, M.C. (2004). Apoyo psicológico en el sufrimiento causado por las pérdidas: el duelo. En R. Bayés (Ed.). Monografías Humanitas 2: Dolor y Sufrimiento en la Práctica Clínica. Barcelona. Fundación Medicina y Humanidades Médicas.

Bonanno, G.A. y Kaltman, S. (2001). The varieties of grief experience. Clinical Psychology Review, 21, 705-734.

Clayton, P.J. (1985). Duelo. En E.S. Paykel (Ed.). Psicopatología do los trastornos afectivos. Madrid. Pirámide.

Echeburúa, E. (2004). Superar un trauma: el tratamiento de las víctimas de sucesos violentos. Madrid. Pirámide.

Echeburúa, E. y Corral, P. (1997). Avances en el tratamiento cognitivoconductual del trastorno de estrés postraumático. Ansiedad y Estrés, 3, 249-264.

Echeburúa, E. y Corral, P. (2001). El duelo norrnal y patológico. En W. Astudillo, E. Clavé y E. Urdaneta (Eds.). Necesidades psicosociales en la terminalidad. San Sebastián. Sociedad Vasca de Cuidados Paliativos.

Echeburúa, E., Corral, P. y Amor, P.J. (2001). Estrategias de afrontamiento ante los sentimientos de culpa. Análisis y Modificación de Conducta, 27, 905-929.

Echeburúa, E., Corral, P. y Amor, P.J. (2005). La resistencia humana ante los traumas y el duelo. En W. Astudillo, A. Casado y C. Mendinueta (Eds.). Alivio de las situaciones difíciles y del sufrimiento 
en la terminalidad. San Sebastián. Sociedad Vasca de Cuidados Paliativos.

Echeburúa, E., Corral, P., Sarasua, B. y Zubizarreta, I. (1996).

Tratamiento cognitivo-conductual del trastorno de estrés postraumático crónico en víctimas de maltrato doméstico: un estudio piloto. Análisis y Modificación de Conducta, 22, 627-654.

Fernández-Montalvo, J. y Echeburúa, E. (1997). Tratamiento cognitivoconductual de la pena mórbida en un caso de ruptura de pareja. Análisis y Modificación de Conducta, 23, 61-83. Hinton, J. (1974). Experiencias sobre el morir. Barcelona. Ariel. Horowitz, M.J., Wilner, N., Marmar, C. y Krupnick, J. (1980). Pathological grief and the activation of latent self images. American Journal of Psychiatry, 137, 1157-1162.

Latiegui, A. (1999). Prevención y tratamiento del duelo patológico. En W. Astudillo, C. Arrieta, C. Mendinueta y I. Vega de Seoane (Eds.). La familia en la terminalidad. Bilbao. Sociedad Vasca de Cuidados Paliativos.

Lazare, A. (1979). Unresolved grief. En A. Lazare (Ed.). Outpatient psychiatry: Diagnostic and treatment. Baltimore. Williams and Wilkens. Lee, C. (1995). La muerte de los seres queridos. Cómo afrontarla y superarla. Barcelona. Plaza Janés.

Mawson, D., Marks, I.M., Ramm, E. y Stern, R. (1981). Guided mourning for morbid grief: A controlled study. British Journal of Psychiatry, $138,185-193$.

Morganett, R.S. (1995). Afrontar la aflicción y pérdida de los seres queridos. En R.S. Morganett. Técnicas de intervención psicológica para adolescentes. Barcelona. Martínez Roca.

Parkes, C.M. (1972). Bereavement: studies of grief in adult life. New York. International University Press.

Pérez Cuesta, M. y Nicuesa, M.J. (1999). Afrontar el proceso de duelo.

En W. Astudillo, A. García de Acilu, C. Mendinueta y C. Arrieta (Eds.). Estrategias para la atención integral en la terminalidad. Bilbao. Sociedad Vasca de Cuidados Paliativos.

Pérez Trenado, M. (1999). El proceso de duelo y la familia. En W. Astudillo, C. Arrieta, C. Mendinueta y I. Vega de Seoane (Eds.). La familia en la terminalidad. Bilbao. Sociedad Vasca de Cuidados Paliativos. 
Rando, T. (1993). Treatment of complicated mourning. Champaign, IL. Research Press.

Raphael, B. (1977). Preventive intervention with the recently bereaved. Archives of General Psychiatry, 34, 1450-1454.

Raphael, B. (1983). The anatomy of bereavement. New York. Basic Books.

Rojas Marcos, L. (2001). Nuestra felicidad. Madrid. Espasa-Calpe. Rojas Marcos, L. (2002). Más allá del 11 de septiembre. La superación del trauma. Madrid. Espasa-Calpe.

Ruiz, J.J. y Cano, J.J. (2002). Manual de psicoterapia cognitiva. Jaén. R\&C Editores.

Savater, F. (1998). El contenido de la felicidad. Madrid. Taurus.

Sireling, L., Cohen, D. y Marks, I.M. (1988). Guided mouming for morbid grief: A controlled replication. Behavior Therapy, 19, 121-132.

Worden, J.W. (1998). El tratamiento del duelo. Asesoramiento psicológico y terapia. Barcelona. Paidós. 